\title{
The independence of the axiom of choice from the Boolean prime ideal theorem*
}

by

\section{J. D. Halpern (Pasadena, Calif.)}

Consider the following statements:

$A C$. For every set $S$ of non-empty sets there is a function $F$ such that $F(x) \in x$ for every $x \in S$.

$E$. There is a function, $F$, on the class of non-empty sets with the property that $F(x) \in x$ for every $x$.

PI. Every Boolean algebra has a prime ideal.

EPI. There is a function $F$ on the class of Boolean algebras such that $F(x)$ is a prime ideal in $x$ for every $x\left(^{1}\right)$.

$R$. There is a non-finite set $S$ of reflexive sets such that every non-empty class $Y$ has an element $x$ with the property $x \cap Y \subset S$ (a reflexive set is $a$ set $x$ satisfying the equation $x=\{x\}$ ).

Let $G$ be the set theory whose axioms are A, B, C, of Gödel [1], suitably modified so that $G$ is a theory with standard formalization in the sense of Tarski [16] whose only non-logical constant is $\epsilon$. Thus $G$ has only one kind of variable; the one place predicates Cls and $M$ are eliminated as follows: Cls $x$ is replaced by $x=x$ and $M(x)$ is replaced by 臬 $y[x \in y]$.

Our theorems are as follows:

THEOREM 1. If $G$ is consistent, $E P I \rightarrow A C$ is not provable in $G+\{R\}$.

Since $E P I \rightarrow P I$ in $G$, we easily obtain

THEOREM 2. If $G$ is consistent, $P I \rightarrow A C$ is not provable in $G+\{R\}$; i.e. $A C$ is independent of $P I$ in $G+\{R\}$.

* The material in this paper is a revision of a part of the author's doctoral dissertation, submitted in the spring of 1962 at the University of California, Berkeley. Theorem 1 in a slightly different form was announced in the Notices of the Amer. Theorem 1 in a slightly different form was announced in the Notices of the Amer.
Math. Soc., Abstract 61T-151. The work was supported, in part, by grant G19286 of Math. Soc., Abstract 61T-151. The

(1) Prof. Tarski has remarked that $P I$ is equivalent to the following: For every set $S$ whose elements are Boolean algebras there is a function $f$ with domain $S$ such that $f(B)$ is a prime ideal in $B$. Thus $E P I$ is a universal version of $P I$ in the same sense that $E$ is the universal version of $A C$. 
By the result of Gödel [1], if $G$ is consistent, $G+\{E\}$ is consistent. By the result of Specker [11], if $G+\{E\}$ is consistent, $G+\{E, R\}$ is consistent. Thus it suffices to prove theorem 1 by finding an interpretation of $G+\{R\}$ in $G+\{E, R\}$. For a discussion of the notion of interpretability, see Tarski [16], p. $20 \mathrm{ff}$. The interpretation that we use is the model $W^{+}$ of Mostowski [6] except for technical changes which have no bearing on the proofs but which allow us to state theorems about $G+\{R\}$ instead of theorems about his system $\mathcal{S}$. Mostowski's model is an interpretation for a set theory which allows urelements, while our interpretation is for a pure set theory; reflexive sets assume the role of urelements. The properties in which we are interested are not affected by this difference. The proofs that the ordering principle and $\sim A . C$ hold in $W^{+}$suffice as proofs that the ordering principle and $\sim A C$ hold in our interpretation. Similarly the proof of EPI in our interpretation suffices as a proof that $E P I$ holds in $W^{+}$. Furthermore, since the ordering principle is a consequence of $P I$ (and hence of $E P I$ ) in both set theories the result of this paper is just a strengthening of Mostowski's result.

$P I$ is equivalent in $G$ to many other interesting theorems in mathematics. In particular it is equivalent to the Stone representation theorem for Boolean algebras (see [12]): the prime ideal theorem for commutative rings with unit (see [10]), the completeness theorem for 1st-order languages (see [2]) and the Tychonoff theorem for $T_{2}$-spaces (see [9]). For other equivalences and consequences, see [2], [4], [9], [13], [14], [15]. In [3] Kelley poses the problem of proving $A C$ from the Tychonoff theorem for $T_{2}$-spaces. Our result gives a negative answer to this question for the theory $G+\{R\}$.

I would like to express my appreciation to Professors Azriel Lévy and Dana Scott. The results mentioned here were obtained while I was working under the direction of Professor Lévy and I am deeply indebted to him for many suggestions. Professor Scott supervised the writing of my thesis, from which this paper is extracted. His comments were heeded, with the result that the presentation was much improved. I am also indebted to him for information concerning Ramsey's theorem at a crucial point in the research. A formulation of the unnumbered theorem in $\$ 4$ in terms of automorphism groups of Boolean algebras which leave a prime ideal invariant, and an application of this formulation to logic are also due to Professor Dana Scott. These will be included in a subsequent paper.

\$1. Preliminaries. We assume the definition of cardinal number as given in Gödel [1]. $N$ will denote the set of finite cardinal numbers. $|X|$ will denote the cardinal number of $X$, for any set $X$. For any class $X$ and any set $Y, X^{Y}$ denotes the class of all functions on $Y$ into $X$. $V$ denotes the universal class. If $n \in N$ and $m \in V^{n}$, we say that $m$ is an $n$-termed sequence and often denote $m(i)$ by $m_{i}$. If $f$ is a function, $\operatorname{Dom} f$ and Rng $f$ denote respectively its domain and range. $f \mid Y$ denotes the restriction of $f$ to the class $Y$. A function which is a 1-1 mapping of its domain onto its domain is said to be a permutation.

For any class $X$, the union of $X$, denoted $\cup X$, is the class of all elements belonging to some member of $X$; the intersection of $X$, denoted $\cap X$, is the class of all elements belonging to every member of $X$; the closure of $X$, denoted $C X$, is the smallest class including $X$ as a subclass and closed in the sense that if $Z \in \mathrm{O} X$ and $Y \in Z$ then $Y \in \mathrm{OX}$. PX is the power set of $X$.

A Boolean algebra $B$ is a 2 -termed sequence whose first term $B_{0}$ is a set and whose second term $B_{1}$ is a partial ordering on $B_{0}$ which satisfies the requirements of a complemented distributive lattice. We will usually write $\leqslant_{B}$ for $B_{1}$. If $X \subseteq \subseteq B_{0}$, we denote by $\operatorname{lnb} X$ and glb $X$ respectively, the least upper bound of $X$ and the greatest lower bound of $X$. If $X$ is finite, $\operatorname{lnb} X$ and glb $X$ exist since $B$ is a lattice. We denote the minimal element of $B$ by $0_{B}$ and the maximal element by $1_{B}$. As part of the definition we assume that $\left|B_{0}\right| \geqslant 2$, which is equivalent to saying that $0_{B}$, $1_{B}$ exist and $0_{B} \neq 1_{B}$. We denote the complement of $b \in B_{0}$ by $-b$. An ideal $I$ in $B$ is a subset of $B_{0}$ satisfying the following three conditions:

(1) $0_{B} \in I$,

(2) $x \in I \wedge y \leqslant_{B} x \rightarrow y \in I$,

(3) $x \in I \wedge y \in I \rightarrow \operatorname{lub}\{x, y\} \in I$.

A prime ideal is a maximal proper ideal or equivalently an ideal with the additional property

(4) $x \in I \leftrightarrow-x \dot{\epsilon} I$.

§ 2. The interpretation $W$. Our development is informal but could be formalized in the theory $G+\{R, E\}$. Let $R$ be the recursively defined ordinal function as follows:

$R(0)$ is a denumerable set of reflexive elements,

$R(\alpha)=\bigcup\{P(R(\beta)): \beta<\alpha\}$ for $\alpha>0$.

Let $U=\bigcup\{R(a) \mid \alpha$ is an ordinal $\}$.

We say that $\varphi$ is an automorphism of $U$ if it is a permutation of $U$ preserving the $\epsilon$-relation, i.e. $x \in y$ if and only if $\varphi(x) \in \varphi(y)$. If $\varphi$ is any permutation of $R(0), \varphi$ induces a unique antomorphism $\varphi^{*}$ of $U$. In the sequel we will confuse $\varphi$ with $\varphi^{*}$. Since for any two permutations $\varphi, \psi$ of $R(0)$

$$
\left(\varphi^{-1}\right)^{*}=\left(\varphi^{*}\right)^{-1} \quad \text { and } \quad(\varphi \psi)^{*}=\varphi^{*} \psi^{*}
$$

and $\varphi^{*}(x)=\varphi(x)$ for $x \in R(0)$, this confusion should not cause any difficulty. 
Let $\boldsymbol{r}$ be an ordering of type $\eta$ (type of the rationals under $<$ ) of the set $R(0)$ and let $G$ be the group of all $\boldsymbol{r}$-preserving permutations of $R(0)$. Let the variable $e$ range over finite subsets of $R(0)$. Let $G[e]$ $=\{\varphi \in G: \varphi(e)=e\} . X \subseteq U$ is said to be e-symmetric if $\operatorname{Rng}(\varphi \mid X)=X$ for every $\varphi \in G[e]$ and $e$ is said to be a support of $X$. $X$ is said to be finitely symmetric if it is $e$-symmetric for some $e$.

Let $W(X)$ be the predicate which asserts that $X$ is finitely symmetric and every element of $\mathrm{C} X$ is finitely symmetric. We will sometimes write $X$ is a $W$-class for $W(X)$ and $X$ is a $W$-set for $W(X) \wedge$ H $Y[W(Y) \wedge X \in Y]$ and we will denote the relativization of a formula $\Phi$ to the predicate $W$ by $\Phi^{W}$. The proofs in [6] may be adapted to show that the relativizations of the axioms of $G$ to the predicate $W$ and $(\sim A C)^{W}$ are theorems of $G+\{R, E\}$. Also it is a simple matter to show that $R^{W}$ is a theorem of $G+\{R, E\}$. It remains to show that $E P I^{W}$ is a theorem of $G+\{R, E\}$. We may think of $W$ as defining a model in the sense of Gödel [1], p. 1 . Employing his definition of absoluteness one easily shows that the $\epsilon$-relation is absolute, that ordered pair, union of a class, intersection of a class are absolute operations, and that the notions of finite class and set are absolute. Thus $X \epsilon^{W} Y$ if and only if $W(X)$ and $W(Y)$ and $X \epsilon Y$ $Z$ is the ordered pair of $X$ and $Y$ in the $W$-sense if and only if $Z$ is the ordered pair of $X$ and $Y$ and $W(X)$ and $W(Y) ; X$ is a finite class in the $W$-sense if and only if $X$ is finite and $W(X) ; X$ is $W$-set and only if $W(X) \wedge B Y[X \in Y]$. With this in mind one easily verifies that $E P I$ under the indicated interpretation becomes the following statement:

There is a function $F$ which is a W-class and whose domain is the class of all W-Boolean algebras (Boolean algebras which are $W$-sets) such that $F(B)$ is a prime ideal in $B$ for every $B$.

§ 3. A combinatorial lemma. In this section we prove a lemma in the theory $G$ which is a corollary of a generalization of Ramsey's theorem, due to R. Rado. The proof given here is due to D. Scott. In preparation we introduce some additional terminology and notation.

A partition of a set $A$ into $k$-parts ( $k$-partition of $A$ ) is a set of cardinality $k$ whose elements are pairwise disjoint and whose union is $A$. To every partition of $A$ there corresponds an equivalence relation on $A$, namely, that equivalence relation whose equivalence classes are the elements of the partition. Similarly, to every equivalence relation on $A$ there is a corresponding partition. If $n \in N, m \in N^{n}$ and $A$ is an $n$-termed sequence of disjoint sets, we denote by $[A]^{m}$ the set of all $Y \subseteq \cup \operatorname{Rng} A$ such that $\left|Y \cap A_{i}\right|=m_{i}$ for $i \epsilon n$. For any $k \epsilon N, k \neq 0$, and any set $X$ we use $[X]^{k}$ to denote the set whose elements are subsets of $X$ with cardinal number $k$. Thus $[X]^{k}=[\{\langle 0, X\rangle\}]^{\{\langle 0, k\rangle\}}$ in terms of the more general notation. Since $N^{n} \cap N=0$ for all $n \in N$ such that $n \neq 0$, there should be no confusion.

For convenience, we state Ramsey's theorem (see [8]): For any $k, l, p \in N$ there is a $q \in N$ such that if $|X| \geqslant q$, then any partition of $[X]^{l}$ into at most $k$ parts has the property that one of the parts include $[Y]^{l}$ for some subset $Y$ of $X$ of cardinality $p$.

The following lemma is contained in the corollary to Theorem 3 in Rado [7]. We will refer to it as Rado's corollary.

LEMma. For all triples $n, m, p$ such that $n \in N, m \in N^{n}, p \in N$, there is a $q \in N$ such that for all $n$-termed sequences $A$ of disjoint sets, if $\left|A_{i}\right| \geqslant q$ for $i \in n$, and $\Delta$ is a 2-partition of $[A]^{m}$, then there is an n-termed sequence $Y$ such that $Y_{i} \subseteq A_{i}$ and $\left|Y_{i}\right|=p$ for $i \epsilon n$, and $[Y]^{m}$ is included in one part of $\Delta$.

Proof. We prove by induction on $n$, that for all $m \in N^{n}$ and $p \in N$, the desired conclusion is obtained.

$n=0$ : The conclusion is racuously satisfied.

$n=1$ : This is just Ramsey's theorem for $k=2$ and $l=m_{0}$.

$n=j+1$ and $j \geqslant 1$ : Let $m \in N^{j+1}$ and $p \in N$. Then $m \mid j \in N^{j}$.

By the induction hypothesis, we obtain a number $q$ which satisfies the lemma for the triple $j, m \mid j, p$. Let $k$ be the number of different 2 -partitions of $[Q]^{m \mid j}$, where $Q$ is any $j$-termed sequence of disjoint sets of cardinality $q$. Let $r$ be a number which satisfies Ramsey's theorem for the triple $k, m_{j}, p$. Let $A$ be any $j+1$-termed sequence of disjoint sets such that

$$
\left|A_{j}\right|=q \text { for } \quad i \in j \text { and }\left|A_{j}\right| \geqslant r .
$$

Let $\Delta$ be any partition of $[A]^{m}$. For elements $x, y \in\left[A_{j}\right]^{m_{j}}$, let $x \equiv_{1} y$ be the relation

$$
\forall z\left[z \in[A \mid j]^{m \mid j} \rightarrow x \cup z \equiv_{\Delta} y \cup z\right]
$$

where $\equiv_{\Delta}$ is the equivalence relation corresponding to $\Delta$. Because of the disjoint condition on $\operatorname{Rng} A$, we see that if

$$
x \in\left[A_{j}\right]^{m_{j}} \text { and } z \in[A \mid j]^{m \mid j} \text {, then } x \cup z \in[A]^{m}
$$

and hence, $x \cup z \equiv_{\Delta} x \cup z$. Thus $\equiv_{1}$ is reflexive. $\equiv_{1}$ is easily seen to be symmetric and transitive, and hence an equivalence relation on $\left[A_{j}\right]^{m_{1}}$. The corresponding partition, $\Delta_{1}$, is seen to have at most $k$ parts (the number of 2 -partitions of $\left.[A \mid j]^{m \mid j}\right)$. By our choice of $\left|A_{j}\right|$, we obtain $X \subseteq A_{j}$ such that $|X|=p$ and $[X]^{m_{j}}$ is included in one part. Since the proof is easily completed if $[X]^{m_{j}}$ is empty, we assume otherwise. Let $y_{0}$ be some element of $[X]^{m s}$. For $x, z \in[A \mid j]^{m \mid j}$ let

$$
a \equiv_{2} z \leftrightarrow x \cup y_{0} \equiv_{\Delta} \approx \cup y_{0} \text {. }
$$


As in the case of $\equiv_{1}$, we verify that $\equiv_{2}$ is an equivalence relation on $[A \mid j]^{m \mid j}$. The corresponding partition $\Delta_{2}$ is seen to be a 2-partition. By the choice of $q$, we obtain a $j$-termed sequence $Y$ such that $Y_{i} \subseteq A_{i}$ and $\left|Y_{i}\right|=p$ for $i \in j$, and $[Y]^{m \mid j}$ is in one part of $\Delta_{2}$. Thus $\left[Y \cup\left\{\left\langle j, y_{0}\right\rangle\right\}\right]^{m}$ is one part of $\Delta$, and from the choice of $X$ this part is the same if we replace $y_{0}$ by any $y \in[X]^{m_{j}}$. Let $Z=[Y \cup\{\langle j, X\rangle\}]^{m}$. Then $Z$ is one part of $A, Z_{i} \subseteq A_{i}$, and $\left|Z_{i}\right|=p$ for $i \in j+1$. Thus $Z$ satisfies the conclusion of the lemma for the sequence $A$ and partition $\Delta$. We have shown that for $A$, and $m$ with domain $j+1$ if $\left|A_{i}\right|=q$ for $i \in j$ and $\left|A_{j}\right| \geqslant r$, then any 2 -partition $A$ has the required property, but then certainly the same holds if $\left|A_{i}\right| \geqslant \max (q, v)$ for $i \epsilon j+1$. Thus the induction step is completed and hence the proof.

§ 4. Proof of $E P I^{W}$. The following lemmas are needed for the proof of $E P I^{W}$. Let $A, B, C$ be subsets of $R(0)$; we say that $A$ divides $B$ and $C$ equally if $A \cap B=A \cap C$ and for every open interval $I$ determined in $R(0)$ by two points of $A$ and the ordering $r,|B \cap I|=|C \cap I|$.

LEMMA 1. If $B, O$ are finite subsets of $R(0)$ and $A$ is a subset of $R(0)$ which divides $B$ and $C$ equally, then there is $\varphi \in G$ such that $\varphi$ leaves $A$ pointwise fixed and $\varphi(B)=C$.

Proof. This is an immediate consequence of the homogeneity of the rationals.

The following two lemmas state easily verified facts about Boolean algebras.

Lenna 2. Let $H$ be a group of automorphisms of a Boolean algebra $B$, and $I$, an ideal closed under $H$. Let $b \in B$. If $J$ is the smallest ideal closed under $H$ which includes $I$ and $\{b\}$ and if $1_{B} \in J$, then there is a finite subset $S \subseteq \underline{H}$ such that $\operatorname{glb}\{\varphi(-b): \varphi \in S\} \in I$.

Lenna 3. If $B$ is a Boolean algebra, $X$ is a finite subset of $B$, and $P$ is the set of all functions $f$ on $X$ such that $f(x) \in\{x,-x\}$, then

$$
\operatorname{lub}\{\operatorname{glb}\{f(x): x \in X\}: f \in P\}=1_{B} .
$$

THEOREMr. If $B$ is a Boolean algebra and $a W$-set, and $e$ is a support of $B$ then there is a prime ideal $I$ of $B$ which is e-symmetric.

Since $B$ is $e$-symmetric, $\{\varphi \mid B: \varphi \in G[e]\}$ is a group of automorphisms of $B$. The theorem asserts that there is a prime ideal of $B$ invariant under this group. The proof is based on the fact that questions concerning the relation between $G[e]$ and $B$ can be reduced to questions concerning $G[e]$ and the supports of elements of $B$.

\section{Proof. Let}

$$
Z=\left\{J: J \text { is an ideal in } B \wedge 1_{B} \xi J \wedge J \text { is } e \text {-symmetric }\right\} .
$$

It is easily verified that $Z$ is a non-empty, partially ordered set under inclusion in which chains have upper bounds, namely their union. Thus by Zorn's form of $A C\left(^{2}\right)$ we obtain an ideal $I$ of $B$ such that $I$ is maximal in $Z$. Suppose $I$ is not prime. Then there exists $b \in B$ such that $b \epsilon I$ and $-b \dot{\epsilon} I$. By Lemma 2 we obtain finite sets $S, T \subseteq G[e]$ such that

$$
\operatorname{glb}\{\varphi(-b): \varphi \in S\} \in I \quad \text { and } \operatorname{gIb}\{\varphi(b): \varphi \in T\} \in I .
$$

Let $|e|=l$. Then $e$ determines $l+1$ open intervals on $R(0)$ relative to the ordering $r$; denote these intervals by $K_{1}, \ldots, K_{l+1}$. Let $g$ be any support of $b$ and let $m$ be the $l+1$-termed sequence such that

$$
\begin{aligned}
& \text { (1) } \quad \varphi \in G[e] \wedge i \in l+1 \rightarrow\left|\varphi(g) \cap K_{i}\right|=m_{i} . \\
& \quad n=\max \{|S|,|T|\}, \quad k=\max \left\{m_{i}: i \in l+1\right\}, \quad p=n \cdot k
\end{aligned}
$$$$
\text { Then } \quad m_{i}=\left|K_{i} \cap g\right|, \quad i \in l+1 .
$$

Let $q$ be a number satisfying Rado's corollary for the triple $l+1$, $m, p$. Since $r$ is dense, we may take subsets $A_{i} \subseteq K_{i}$ such that $\left|A_{i}\right|=q$ for $i \in l+1$. Let $A$ be the sequence so defined. Let

$$
H=\left\{\langle a, x\rangle: \mathbb{H} \varphi\left[\varphi \in G[e] \wedge \varphi(b)=x \wedge \varphi(g)-e=a \wedge a \in A^{[m]}\right]\right\} .
$$

$H$ is a function since $g$ is a support of $b$. From Lemma 1 it follows that $\operatorname{Dom} H=A^{[m]}$ and a fortiori since $[A]^{m}$ is finite, $\operatorname{Rng} H$ is finite. Let $f$ be any function on Rng $H$ such that $f(x)=x$ or $f(x)=-x$ for each $x \in \operatorname{Rng} H$. To show that $1_{B} \in I$ we need only show that glb $\{f(x): x \in \operatorname{Rng} H\}$ $\epsilon I$ and then apply lemma 3 . To this end let

$$
\Delta_{1}=\{a: f(H(a))=H(a)\}, \quad \Delta_{2}=\{a: f(H(a))=-H(a)\} ;
$$

$\left\{\Delta_{1}, \Delta_{2}\right\}$ constitutes a 2 -partition of $[A]^{m}$. Thus by Rado's corollary we get a function $Y$ on $l+1$ such that

$$
Y_{i} \subset A_{i} \text { and } \quad\left|Y_{i}\right|=p \text { for } i \epsilon l+1
$$

and $[Y]^{m} \subseteq \Delta_{1}$ or $[Y]^{m} \subseteq \Delta_{2}$

Case 1. $[Y]^{m}\left(A_{1}\right.$. From (1) it follows that $\left|K_{i} \cap \bigcup\left\{\varphi(g): \varphi \in T^{T}\right\}\right|$ $\leqslant m_{i} \cdot|T|$. Since $\left|Y_{i}\right|=p \geqslant m_{i} \cdot|T|$, we have by lemma 1 , the existence of a $\psi \in G[e]$ such that $\psi(\bigcup\{\varphi(g): \varphi \in T\}) \subseteq e \cup \cup \operatorname{Rng} Y$. Thus $\psi \varphi(g)$ $C e \cup \cup \operatorname{Rng} I$ and hence

$$
\psi \varphi(g) \cap K_{i} \subseteq Y_{i}, \quad i \in l+1, \varphi \in T
$$

(') Prof. Scott has remarked that appeal to Zorn's lemma here can be replaced by an appeal to $P I$. 
From (1) it follows that

$$
\psi \varphi(g)-e \in[Y]^{m} \text { for } \varphi \in T .
$$

Since $\psi \varphi \in G[e]$ and $[Y]^{m} \subseteq \Delta_{1}$, it follows that $\psi \varphi(b) \in \operatorname{Rng} H$ and $f(\psi \varphi(b))=\psi \varphi(b)$ for $\varphi \in T$. Thus

$$
\operatorname{glb}\{f(x): x \in \operatorname{Rng} H\} \leqslant_{B} \operatorname{glb}\{\psi \varphi(b): \varphi \in T\}=\psi \operatorname{glb}\{\varphi(b): \varphi \in T\} .
$$

Since $\psi \in G[e], \operatorname{glb}\{\varphi(b): \varphi \in T\} \in I$, and $I$ is $e$-symmetric, we conclude $\operatorname{glb}\{f(x): x \in \operatorname{Rng} H\} \in I$.

Case 2. $[Y]^{m} \subseteq \Delta_{2}$. Proceeding as in Case 1 with $T$ replaced by $S$, we obtain the existence of $\psi \in G[e]$ such that

$$
\psi \varphi(g)-e \in[Y]^{m} \quad \text { for } \quad \varphi \in S .
$$

As in Case 1, we infer that $\psi \varphi(b) \in \operatorname{Rng} H$ and

Thus

$$
f(\psi \varphi(b))=-\psi \varphi(b) \quad \text { for } \quad \varphi \in S .
$$

$\operatorname{glb}\{f(x): x \in \operatorname{Rng} H\} \leqslant_{B} \operatorname{glb}\{-\psi \varphi(b): \varphi \in S\}=\psi \operatorname{glb}\{\varphi(-b): \varphi \in S\}$.

Since $\operatorname{glb}\{\varphi(-b): \varphi \in S\} \in I$, it follows as in Case 1 that

$$
\operatorname{glb}\{f(x): x \in \operatorname{Rng} H\} \in I \text {. }
$$

Thus $\operatorname{glb}\{f(x): x \in \mathrm{Rng} H\}$ for any function $f$ on Rng $H$ such that $f(x) \in\{x,-\infty\}$. Since Rng $H$ is finite and $I$ is an ideal, it follows from Lemma 3 that $1_{B} \in I$ which is a contradiction. Hence $I$ is prime, q.e.d.

LEMar 4. The intersection of all supports of a $W$-class $X$ is itself a support of $X$; we denote it by $e_{X}$.

Proof. This is a direct consequence of the homogeneity of the ordering $\boldsymbol{r}$ and the definition of $e$-symmetric. The proof is carried out in detail in Mostowski [6].

LEMMA 5. If $X$ is a $W$-class, $\varphi \in G$, and $\varphi(X)=X$ then $\varphi\left(e_{X}\right)=e_{X}$.

Proof. Suppose $\varphi(X)=X$ and $\varphi\left(e_{X}\right) \neq e_{X}$. Since $e_{X}$ is finite and $\varphi$ is a permutation of $R(0)$, it follows that $e_{X} \nsubseteq \varphi\left(e_{X}\right)$. But $\varphi\left(e_{X}\right)$ is a support of $X$; for suppose $\psi \epsilon G\left[\varphi\left(e_{X}\right)\right]$, then $\psi \varphi^{-1}\left(e_{X}\right)=e_{X}$ and hence $\psi \varphi^{-1}(X)=X$. But $\varphi^{-1}(X)=X$ and hence $\psi(X)=X$. Thus $\varphi\left(e_{X}\right)$ is a support of $X$, contradicting the definition of $e_{X}$.

LEMMA 6. If $X$ is e-symmetric for some finite set $e$ and every element of $X$ is a $W$-set then $W(X)$.

The proof is immediate from the definition of $W$.
Proof of $E P I^{W}$. For every $W$-Boolean algebra $B$, let

$$
[B]=\{Y: \mathbb{H} \varphi \in G[\varphi(B)=Y]\} .
$$

$[B]$ is a set since the rank of every element of $[B]$ is the same as $B$. Also every element of $B$ is easily seen to be a $W$-Boolean algebra. Distinct $[B]$ 's are disjoint since $G$ is a group. Let $F^{*}$ denote the function whose existence is asserted by axiom $E$. For every $W$-Boolean algebra $B$, let $I(B)=\left\{I: I\right.$ is a prime ideal of $B$ and $e_{B}$ is a support of $\left.I\right\}$. From the theorem it follows that $I(B)$ is non-empty. Let

$$
K=\left\{F^{*}([B]): B \text { is a } W \text {-Boolean algebra }\right\}
$$

and let $F_{1}=\left\{\langle B, J\rangle: B \in K \wedge F^{*}(I(B))=J\right\}$.

Let $F=\left\{X:\right.$ H $Y$ H $\varphi\left[Y \in F_{1} \wedge \varphi \in G \wedge \varphi(Y)=X\right\}$. $F$ is easily seen to be a class of ordered pairs $\langle Z, V\rangle$ such that $Z$ is a $W$-Boolean algebra and $V$ is a prime ideal of $Z$. $e_{Z}$ is a support of $V$, and hence $V$ is a $W$-set by Lemma $6 . F$ has the empty set as a support and thus is a $W$-class by Lemma $6 . F$ is a function because of Lemma 5 and the disjointedness of the $[B]$ 's. The domain of $F$ is easily seen to be the class of $W$-Boolean algebras. Thus $F$ satisfies the desired conditions.

\section{References}

[1] K. Gödel, The consistency of the continuum hypothesis, Annals of Math. Studies 3, Princeton 1940 .

[2] L. A. Henkin, Matemathematical theorems equivalent to the prime ideal heorems for Boolean algebras, Bull. Amer. Nath. Soc. 60 (1954), pp. 387-388.

[3] J. L. Kelley, The Tychonoff product theorem implies the axiom of choice, Fund. Math. 37 (1950), pp. 75-76.

[4] J. Łoś and C. Ryll-Nardzewski, Effectiveness of the representation theory for Boolean algebras, ibidem 41 (1954-55), pp. 49-56.

[5] - On the application of Tychonoff's theorem in mathematical proofs, ibidem 38 (1951), pp. 233-237.

[6] A. Mostowski, Über die Unabhängigkeit des Wohlordnungssatz vom Ordnungsprinzip, ibidem 32 (1939), pp. 201-252.

[7] R. Rado, Direct ilecomposition of partitions, J. Lond. Math. Soc. 29 (1954), pp. $71-83$.

[8] F. P. Ramsey, On a problem of formal logic, Proc. Lond. Math. Soc. 30 (1929), Ser. 2, p. 264-286.

[9] H. Rubin, D. Scott, Some topological theorems equivalent to the Boolean prime ideal theorem, Bull. Amer. Math. Soc. 60 (1954), p. 389.

$[10] \mathrm{D}$. Scott, Prime ideal theorems for rings, lattices, and Boolean algebras, ibidem 60 (1954), p. 390

[11] E. Specker, Zur Axiomatil der Mengenlehre (Fundierungs- und Auswahlaxiom), Zeitschrift f. Math. Logik und Grundlagen d. Math. 3 (1957), pp. 173-210.

[12] M. H. Stone, The theory of rerpesentations for Boolean algebras, Trans. of Amer. Math. Soc. 40 (1936), pp. 37-111.

Fundamenta Mathematicae, T. LV 
[13] A. Tarski, Prime ideal theorems for Boolean algebras and the axiom of choice, Bull. Amer. Math. Soc. 60 (1954), pp. 390-391.

[14] - Prime ideal theorems for set algebras and ordering principles, ibidem 60 (1954), p. 391.

[15] - Prime ideal theorems for set algebras and the axiom of choice, ibidem 60 (1954), p. 391.

[16] A. Tarski, A. Mostowski, R. Robinson, Undecidable Theories, Amster. dam 1953.

CALIFORNIA INSTITUTE OF TECHNOLOGY

Reģu par la Rédaction le 2\%.2.1963

\section{Sequentially pointwise continuous linear functionals}

by

1. Introduction. If $L$ is a linear space of real-valued functions on a non-empty set $X$ and $\varphi$ is a linear functional on $L$ which is continuous with respect to pointwise convergence on $X$ of nets in $L$, then it is an immediate consequence of the theory of duality in linear spaces that there are points $x_{1}, \ldots, x_{n}$ in $X$ and real numbers $\lambda_{1}, \ldots, \lambda_{n}$ such that

$$
\varphi(f)=\sum_{i=1}^{n} \lambda_{i} f\left(x_{i}\right)
$$

for all $f$ in $L$. If, however, we ask under what conditions every linear functional on $L$ which is continuous with respect to pointwise convergence on $X$ of sequences in $L$ (sequentially or $\sigma p$-continuous) is of this form then the problem is much more complicated. For example, Mrówka [5] constructs a positive $\sigma p$-continuous linear functional on a linear space of continuous real-valued functions on the unit interval, $[0,1]$, of the reals which is far from being of this form. On the other hand, he shows [3] that if $L$ is the linear space $C(X)$ of all continuous real-valued functions on a completely regular Hausdorff space $X$, then a necessary and sufficient condition is that $X$ be real-compact (i.e. complete with respect to the weak uniformity induced by $C(X))$. In [4] and [5] he gives necessary and sufficient conditions for certain algebras of real-valued functions and linear lattices of bounded real-valued functions.

The present paper is chiefly concerned with the case where $L$ is a linear lattice. For such an $L$ we prove, (a) that the non-trivial $\sigma p$-continuous linear lattice $\left(^{1}\right)$ functionals are, up to a positive multiple, in (1-1) correspondence with the proper prime $\sigma$-filters of zero sets on $X$ (these are precisely the $\sigma$-filters of zero sets which are maximal proper filters of zero sets) (Theorem 1); and (b) that for any $\sigma p$-continuous linear functional, $\varphi$, on $L$ there is a unique class, $\delta_{\varphi}$, of non-trivial $\sigma p$-continuous 